\title{
Microfluidic Behavior of Ternary Mixed Carrier Solvents Based on the Tube Radial Distribution in Triple-Branched Microchannels in a Microchip
}

\author{
Naoya Jinno, Masahiko Hashimoto, Kazuhiko Tsukagoshi" \\ Department of Chemical Engineering and Materials Science, Faculty of Science and Engineering, Doshisha University, Kyotanabe, \\ Japan. \\ Email: *ktsukago@mail.doshisha.ac.jp
}

Received March $1^{\text {st }}, 2012$; revised March 21 ${ }^{\text {st }}, 2012$; accepted April $8^{\text {th }}, 2012$

\begin{abstract}
Microfluidic behavior of ternary mixed carrier solvents of water-acetonitrile-ethyl acetate (2:3:1 volume ratio) was examined by use of a microchip incorporating microchannels in which one wide channel was separated into three narrow channels, i.e., triple-branched microchannels. When the ternary carrier solution containing the fluorescent dyes, hydrophobic perylene (blue) and relatively hydrophilic Eosin Y (green), was fed into the wide channel under laminar flow conditions, the carrier solvent molecules or fluorescence dyes were radially distributed in the channel, forming inner (organic solvent-rich major; blue) and outer (water-rich minor; green) phases in the wide channel. And then, in the narrow channels, perylene molecules mostly appeared to flow through the center narrow channel and Eosin Y, which is distributed in the outer phases in the wide channel, flowed through the both side narrow channels. A metal ion, $\mathrm{Cu}(\mathrm{II})$ as a model, dissolved in the ternary mixed carrier solution was also examined. $\mathrm{The} \mathrm{Cu}(\mathrm{II})$ showed fluidic behavior, transferring from the homogeneous carrier solution to the water-rich solution in the side narrow channels through the triple-branched microchannels.
\end{abstract}

Keywords: Triple-Branched Microchannels; Ternary Mixed Solvents; Fluorescence Dyes; Metal Ion; Tube Radial Distribution Phenomenon (TRDP)

\section{Introduction}

The development of micro total analysis systems that include a microchip or microfluidic device technology is an interesting aspect of analytical chemistry [1,2]. Microfluidics exhibit various types of fluidic behavior of solvents in a microchannel. The microfluidic behavior has been examined by varying the channel configuration and flow rate of the solvents, by using aqueous-organic solvent mixtures, and by introducing specific obstacles into the microchannel [3-5]. The fluidic behavior of the solvents in the microchannel is related to the mixing, separation, diffusion, and reaction of the solutes. Information regarding the flow pattern of the solvents is important and useful to design microreactors or micro total analysis systems $[6,7]$.

Recently, we reported that when ternary mixed solvents of water-hydrophilic/hydrophobic organic solvents are fed into a microspace, such as microchannels in a microchip or capillary tubes, the solvent molecules are radially distributed under laminar flow conditions. This is called

"Corresponding author. the "tube radial distribution phenomenon" (TRDP) [811]. In TRDP, in an organic solvent-rich carrier solution, the organic solvent-rich major phase is formed around the center of the microspace as an inner phase, while the water-rich minor phase is formed near the inner wall as an outer phase. On the other hand, in the water-rich carrier solution, the water-rich major phase is formed as an inner phase and the organic solvent-rich minor phase as an outer phase. TRDP forms a specific liquid-liquid interface, which is not static but kinetic, in a microspace.

Until now, TRDP has been investigated by use of mainly capillary tubes and straight single line microchannels [8-11]. The triple-branched microchannels in which one wide channel was separated into three narrow channels in a microchip was designed and manufactured in our previous study to conform TRDP, where the ternary mixed carrier solution was fed from the three narrow channels to the one wide channels [8]. In this study microfluidic behavior of the ternary mixed carrier solution in the triple-branched microchannels was examined with the reverse flow direction against the previous paper [8], i.e., 
the flow direction form the one wide channel to the three narrow channels. By the experiments, we could observe unique fluidic behavior of the solvents and solutes in the microchannels based on TRDP. The preliminary results reported in a communication previously [12].

\section{Experimental}

\subsection{Reagents and Materials}

Water was purified with an Elix 3 UV system (Millipore Co., Billerica, MA). All reagents used were obtained commercially and were of analytical grade. Perylene, Eosin $\mathrm{Y}$, acetonitrile, ethyl acetate, and $\mathrm{Cu}$ (II) chloride were purchased from Wako Pure Chemical Industries, Ltd. (Osaka, Japan). Orange $G$ was purchased from Nacalai Tesque, Inc. (Kyoto, Japan). A microchip made of quartz incorporating microchannels was manufactured with Microchemical Technology (Kanagawa, Japan). Figure 1 illustrates the setup of the microchip in the microchip holder (Figure 1(a)) and the enlarged view of the microchip incorporating the triple-branched microchannels (Figure 1(b)). As shown in Figure 1(b)), one wide channel $(300 \mu \mathrm{m}$ wide $\times 40 \mu \mathrm{m}$ deep $)$ denoted as channel $\mathrm{W}$ was separated into three narrow channels (each 100 $\mu \mathrm{m}$ wide $\times 40 \mu \mathrm{m}$ deep) designated as channels N1-N3.

\subsection{Fluorescence Microscope Equipped with a CCD Camera System}

A microchip incorporating the microchannels was set up for the fluorescence microscope-CCD camera system. Fluorescence in the microchannel was monitored using a fluorescence microscope (BX51; Olympus, Tokyo, Japan) equipped with an Hg lamp, a filter (U-MWU2, ex. 330 -

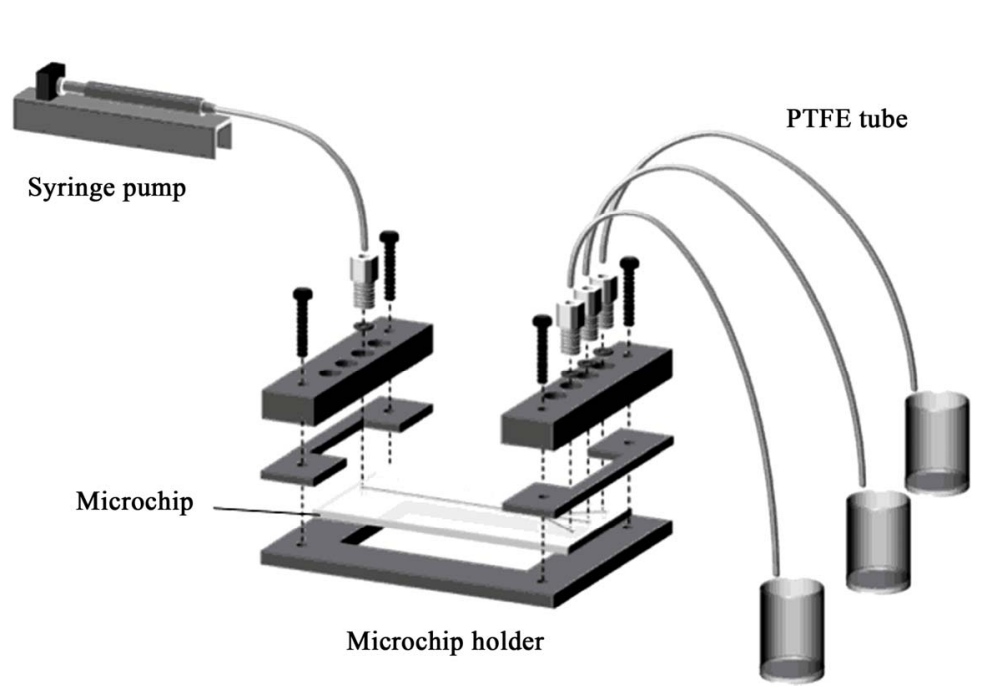

(a)
$385 \mathrm{~nm}$, em. $>420 \mathrm{~nm}$ ), and a CCD camera (JK-TU53H). The ternary mixed solvents of the water-acetonitrileethyl acetate mixture (3:8:4 volume ratio) contained 0.1 $\mathrm{mM}$ perylene and $1 \mathrm{mM}$ Eosin Y. The carrier solution was fed into the microchannels using a microsyringe pump.

\subsection{Cu(II) Analysis}

The homogenous carrier solution of the water-acetonetrile-ethyl acetate mixture (2:3:1 volume ratio) including $2.0 \mathrm{mM} \mathrm{Cu}(\mathrm{II})$ was fed into channel $\mathrm{W}$ at a definite flow rate. $100 \mu \mathrm{L}$ of the solution in channels N1-N3 was collected through polytetrafluoroethylene (PTFE) tubes into the corresponding vessel. The solution was dried under a vacuum, and $0.5 \mathrm{M}$ ammonia solution $(100 \mu \mathrm{L})$ was added to the residue for flow absorption measurement at $600 \mathrm{~nm}$ (modified SPD-6AV spectrophotometric detector, Shimadzu Co., Kyoto, Japan).

\section{Results and Discussion}

\subsection{Fundamental Experiments for TRDP in a Microchannel}

Microchips with single-branched microchannels having widths of $100,200,300$, and $400 \mu \mathrm{m}$ and identical channel depth of $40 \mu \mathrm{m}$ were also manufactured. The influences of channel width on TRDP in the microchannels were examined with fluorescent dyes, perylene and Eosin Y, dissolved in an organic solvent-rich carrier solution (water-acetonitrile-ethyl acetate; 3:8:4 volume ratio). The fluorescence images of the dyes in the solution fed into the microchannels were observed with the fluorescence microscope-CCD camera system (Figure 2). The flow

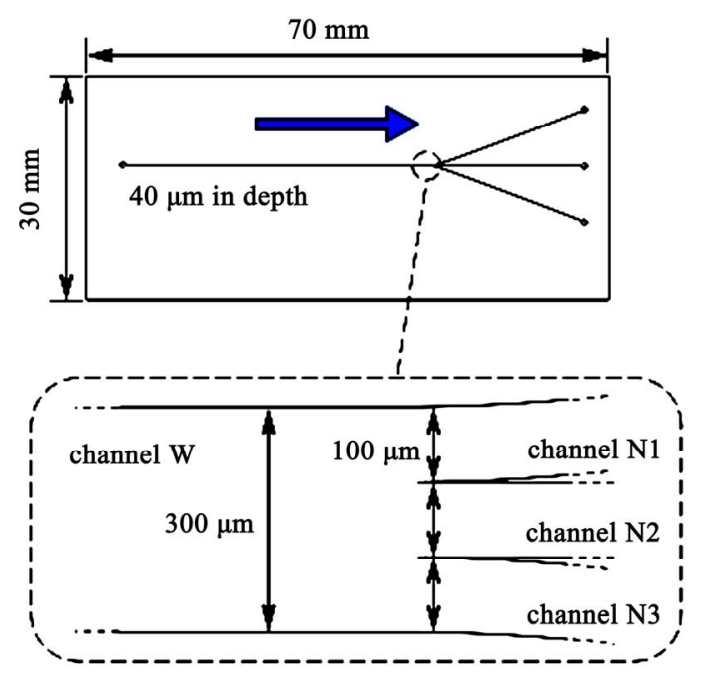

(b)

Figure 1. Schematic representation of (a) setup of the microchip in the microchip holder and (b) the enlarged view of the microchip incorporating triple-branched microchannels. 
rates in all microchannels (100 - $400 \mu \mathrm{m}$ width) were adjusted to achieve the same average linear velocity of $25 \mathrm{~cm} \cdot \mathrm{min}^{-1}$.

It is evident from Figure 2 that TRDP was observed in all microchannels regardless of the channel width. Hydrophobic perylene molecule (blue) was distributed around the center of the channel away from the side inner walls of the channel, while relatively hydrophilic Eosin Y molecule (green) was distributed near the side inner walls of the channel. That is, the major inner phase (organic solvent-rich phase) and the minor outer phase (water-rich phase) were formed in the microchannels of 100 - $400 \mu \mathrm{m}$ width.

\subsection{Effects of Flow Rates on TRDP in Triple-Branched Microchannels}

The effects of flow rate on TRDP in the triple-branched microchannels were examined with an organic solventrich carrier solution (water-acetonitrile-ethyl acetate, 3:8:4 volume ratio) containing Orange $G$ by using the bright-field microscope-CCD camera system. The Orange G-containing carrier solution was fed at the flow rates of $2.0-50.0 \mu \mathrm{L} \cdot \mathrm{min}^{-1}$. The images are shown in Figure 3. The hydrophilic Orange G molecule was distributed near the channel side inner walls, and the interface between the water-rich outer phase and the organic solvent-rich inner phase was distinguished because of the color of Orange G (Figure 3). The TRDP was for the first time observed with a bright-field microscope-CCD camera system in our TRDP investigation. The system was confirmed to be useful for TRDP observation in a similar way to fluorescence system. At the low flow rates of 2.0 and $5.0 \mu \mathrm{L} \cdot \mathrm{min}^{-1}$, the liquid-liquid interface was unstable, considerably changed, or appeared to instantaneously disappear, while the stability was observed at the flow rates higher than $5 \mu \mathrm{L} \cdot \mathrm{min}^{-1}$.

Thus, flow rates higher than $5 \mu \mathrm{L} \cdot \mathrm{min}^{-1}$ created a stable liquid-liquid interface in the triple-branched microchannels under the present conditions.
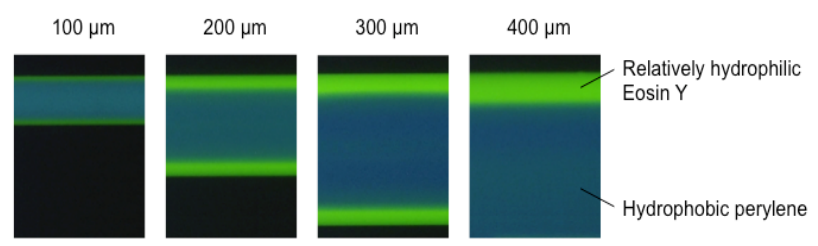

Figure 2. Fluorescence images of fluorescent dyes dissolved in a ternary mixed carrier solvent in microchannels with channel widths of $100,200,300$, and $400 \mu \mathrm{m}$ and identical channel depth of $40 \mu \mathrm{m}$. Conditions: Carrier, water-acetonitrile-ethyl acetate $(3: 8: 4 \mathrm{v} / \mathrm{v} / \mathrm{v})$ mixture including 0.1 $\mathrm{mM}$ perylene and $1 \mathrm{mM}$ Eosin $\mathrm{Y}$; and flow rates, 1, 2, 3, and $4 \mu \mathrm{L} \cdot \mathrm{min}^{-1}$ for microchannel widths of $100,200,300$, and $400 \mu \mathrm{m}$, respectively.

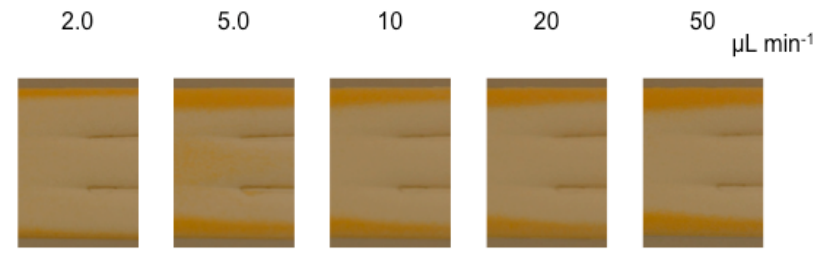

Figure 3. Effects of flow rates of the carrier solution on TRDP. Conditions: Carrier, water-acetonitrile-ethyl acetate (3:8:4 v/v/v) mixture containing $2 \mathrm{mM}$ Orange $G$ and flow rate, 2.0 - $50 \mu \mathrm{L} \cdot \mathrm{min}^{-1}$ for channel $W$.
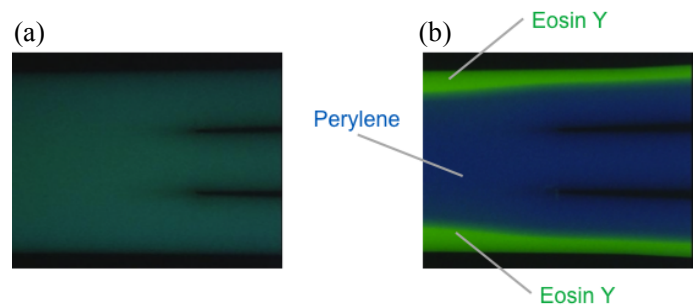

Figure 4. Fluorescence images of fluorescent dyes dissolved in a mixed carrier solvent in triple-branched microchannels. (a) Water-acetonitrile $(1: 4 \mathrm{v} / \mathrm{v})$ and (b) water-acetonetrileethyl acetate $(3: 8: 4 \mathrm{v} / \mathrm{v} / \mathrm{v})$ mixtures. Conditions: Carrier, aqueous-organic mixtures including $0.1 \mathrm{mM}$ perylene and 1 mM Eosin Y; and flow rate, $6.3 \mu \mathrm{L} \cdot \mathrm{min}^{-1}$ for channel $W$.

\subsection{Fluorescence Images of the Dyes Based on TRDP in Triple-Branched Microchannels}

The microchip incorporating triple-branched microchannels was shown in Figure 1(b) with their measurements. The organic solvent-rich carrier solution (water-acetonitrileethyl acetate, 3:8:4 volume ratio) containing Eosin Y and perylene was fed into channel $\mathrm{W}$ of the microchip at the flow rate of $6.3 \mu \mathrm{L} \cdot \mathrm{min}^{-1}$. The mixture carrier solution excluding ethyl acetate (water-acetonitrile, 1:4 volume ratio) but containing the dyes was also fed into the channel as a reference. Figure 4 shows the fluorescence images of the dyes in the triple-branched microchannels of the microchip. Figures 4(a) and (b) were obtained with the water-acetonitrile mixture as a reference and the water-acetonitrile-ethyl acetate mixture as ternary mixed solvents, respectively.

The distribution behavior of the dyes, i.e., TRDP, was not observed in the image of Figure 4(a) with the wateracetonitrile mixture. On the contrary, the image of Figure 4(b) with the ternary solvent mixture shows TRDP, in which the hydrophobic perylene molecule (blue) was distributed around the center of channel W away from the channel side inner walls, while relatively hydrophilic Eosin Y molecule (green) was distributed near the channel side inner walls. The distribution phenomena of the ternary mixed carrier solvents fed into the microchannels was evident in the wide channel. And then, perylene molecules 
mostly appeared to flow through the center channel (channel N2) and Eosin Y, which is distributed in the outer phases in channel $\mathrm{W}$, flowed through channels N1 and N3, as shown in Figure 4(b).

\subsection{Distribution of Eosin $Y$ Based on TRDP in Triple-Branched Microchannels}

The homogenous carrier solution of the water-acetonetrile-ethyl acetate mixture (3:8:4 volume ratio) including $0.5 \mathrm{mM}$ Eosin $\mathrm{Y}$ was fed into channel $\mathrm{W}$ at a flow rate of $10 \mu \mathrm{L} \cdot \mathrm{min}^{-1} .100 \mu \mathrm{L}$ of the solution in channels N1-N3 was collected through PTFE tubes into the corresponding vessel. The solution was subjected to flow absorption measurement at $517 \mathrm{~nm}$ (modified SPD-6AV spectrophotometric detector, Shimadzu Co., Kyoto, Japan). Eosin $\mathrm{Y}$ concentrations in the center channel (channel N2) and the two side channels (channels N1 and N3) were $0.36,0.59$, and $0.58 \mathrm{mM}$, respectively (Figure 5). The concentrations were averages for five measurements. The experimental data indicated that relatively hydrophilic Eosin $\mathrm{Y}$ was distributed from the homogeneous carrier solution to the water-rich solution in the side narrow channels rather than the organic solvent-rich solution in the central narrow channel. The distribution of the fluorescent dyes in the triple-branched microchannels might suggested the possibility of extraction or separation for some solutes by utilizing the phase interface formed by TRDP in these channels.

\subsection{Distribution of $\mathrm{Cu}$ (II) Based on TRDP in Triple-Branched Microchannels}

Distribution of $\mathrm{Cu}(\mathrm{II})$ was examined in the triplebranched microchannels by utilizing the kinetic liquidliquid or aqueous-organic interface formed by TRDP. First, the calibration curve of $\mathrm{Cu}$ (II) was examined in a manner similar to that described in the experimental section (Sec 2.3). $\mathrm{Cu}(\mathrm{II})$ was determined over the concentration range of $0.5-10 \mathrm{mM}$ with good linearity $\left(\mathrm{R}^{2}=\right.$ 0.999).

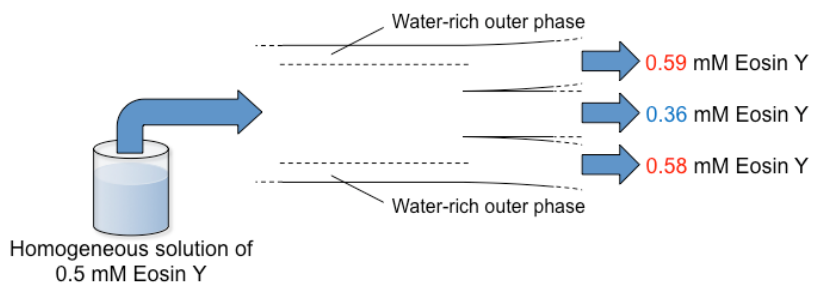

Figure 5. Distribution of Eosin $Y$ from the homogeneous ternary mixed solution into the outer phases in the side narrow channels by TRDP. Conditions: Carrier, wateracetonitrile-ethyl acetate $(3: 8: 4 \mathrm{v} / \mathrm{v} / \mathrm{v})$ mixture; flow rate, 10 $\mu \mathrm{L} \cdot \mathrm{min}^{-1}$ for channel $\mathrm{W}$; Eosin $\mathrm{Y}$ concentration in the homogeneous solution, $0.50 \mathrm{mM}$.
The homogenous carrier solution of the water-acetonitrile-ethyl acetate mixture (2:3:1 volume ratio) including $2.0 \mathrm{mM} \mathrm{Cu}(\mathrm{II})$ was fed into channel $\mathrm{W}$ at the flow rate of $40 \mu \mathrm{L} \cdot \mathrm{min}^{-1} .100 \mu \mathrm{L}$ of the solution in channels N1-N3 was collected with PTFE tubes into the corresponding vessel. $\mathrm{Cu}(\mathrm{II})$ concentrations in the center wide channel (channel N2) and the two side channels (channels N1 and N3) were 0.8, 2.5, and $2.7 \mathrm{mM}$, respectively (Figure 6(a)). The concentrations were averages for five measurements. As a reference, $\mathrm{Cu}(\mathrm{II})$ concentrations were examined with the water-acetonitrile (2:3 volume ratio) carrier solution in a similar manner. $\mathrm{Cu}(\mathrm{II})$ concentrations in channels N1-N3 were 2.1, 2.1, and 2.0 $\mathrm{mM}$, respectively (Figure 6(b)). The experimental data indicated that $\mathrm{Cu}$ (II) was extracted to the water-rich solution in the side narrow channels from the homogeneous solution by the formation of a phase interface in the microchannel; we refer to this process as tube radial distribution extraction (TRDE).

Moreover, we examined the $\mathrm{Cu}(\mathrm{II})$ distribution by TRDE at other flow rates of 32 and $63 \mu \mathrm{L} \cdot \mathrm{min}^{-1}$ in channel W. At $32 \mu \mathrm{L} \cdot \mathrm{min}^{-1}, \mathrm{Cu}(\mathrm{II})$ concentrations in the center channel (channel N2) and the two side channels (channels $\mathrm{N} 1$ and $\mathrm{N} 3$ ) were $0.8,3.0$, and $2.9 \mathrm{mM}$, whereas, at $63 \mu \mathrm{L} \mathrm{min}{ }^{-1}$, those were $1.1,2.9$, and $2.7 \mathrm{mM}$, respectively. Comparing with the $\mathrm{Cu}(\mathrm{II})$ concentration, $2.0 \mathrm{mM}$, in the homogeneous carrier solution, the $\mathrm{Cu}(\mathrm{II})$ concentration in the solution collected from the center channel (channel N2) decreased, while that from the two side channels (channels $\mathrm{N} 1$ and $\mathrm{N} 3$ ) increased at every

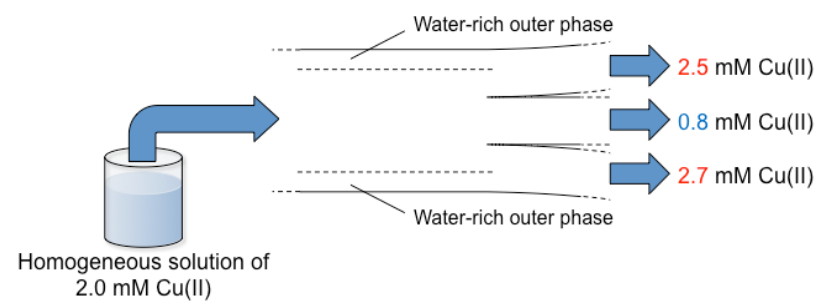

(a)

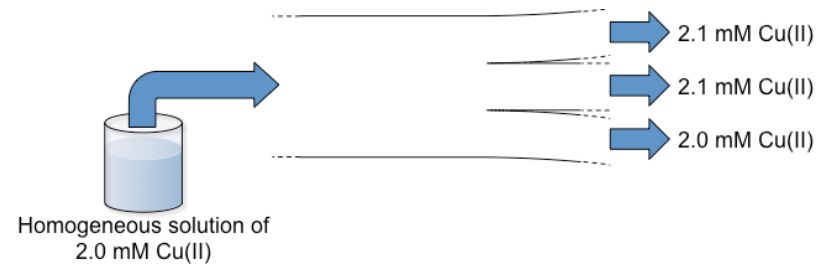

(b)

Figure 6. Distribution of $\mathrm{Cu}(\mathrm{II})$ in triple-branched microchannels. (a) Water-acetonitrile-ethyl acetate $(2: 3: 1 \mathrm{v} / \mathrm{v} / \mathrm{v})$ mixture and (b) water-acetonitrile $(2: 3 \mathrm{v} / \mathrm{v})$ mixture carrier solution. Conditions: Flow rate, $40 \mu \mathrm{L} \cdot \mathrm{min}^{-1}$ for channel W and $\mathrm{Cu}(\mathrm{II})$ concentration in the homogeneous solution, 2.0 mM. 
flow rates. The obtained data showed that TRDE occurred in the triple-branched microchannels of the microchip.

\section{Conclusion}

TRDP has been investigated by use of mainly capillary tubes and straight single line microchannels. The TRDP of the ternary mixed carrier solution in the triplebranched microchannels was observed with fluorescence microscope-CCD camera system. Consequently, perylene molecules mostly appeared to flow through the center narrow channel and Eosin Y flowed through channels in the side narrow channels. The TRDP was also observed through bright-light microscope-CCD camera system. We tried to apply the phase interface formed by TRDP, i.e., inner and outer phases, to the distribution or extraction procedure of $\mathrm{Cu}(\mathrm{II})$ in the triple-branched microchannels. This process will be helpful for investigating separation and extraction in a microfluidic device.

\section{Acknowledgements}

This work was supported by a Grant-in-Aid for Scientific Research (C) from the Ministry of Education, Culture, Sports, Science, and Technology, Japan. This work was also supported by "Advanced Study for Integrated Particle Science, and Technology," Strategic Development of Research Infrastructure for Private Universities, the Ministry of Education, Culture, Sports, Science, and Technology, Japan.

\section{REFERENCES}

[1] K. Ueno, F. Kitagawa and N. Kitamura, "One-Step Electrochemical Cyanation Reaction of Pyrene in Polymer Microchannel-Electrode Chips," Bulletin of the Chemical Society of Japan, Vol. 77, No. 7, 2011, pp. 1331-1338. doi:10.1246/bcsj.77.1331

[2] D. R. Reyes, D. Iossifidis, P. A. Auroux and A. Manz, "Micro Total Analysis Systems. 1. Introduction, Theory, and Technology," Analytical Chemistry, Vol. 74, No. 12, 2002, pp. 2623-2636. doi:10.1021/ac0202435

[3] Y. Kikutani, H. Hisamoto, M. Tokeshi and T. Kitamori, "Micro Wet Analysis System Using Multi-Phase Laminar Flows in Three-Dimensional Microchannel Network,"
Lab on a Chip, Vol. 4, No. 4, 2004, pp. 328-332. doi:10.1039/b400233d

[4] A. Hibara, M. Tokeshi, K. Uchiyama, H. Hisamoto and T. Kitamori, "Integrated Multilayer Flow System on a Microchip," Analytical Sciences, Vol. 17, No. 1, 2001, pp. 89-93. doi:10.2116/analsci.17.89

[5] N. Kaji, Y. Okamoto, M. Tokeshi and Y. Baba, "Nanopillar, Nanoball, and Nanofibers for Highly Efficient Analysis of Biomolecules," Chemical Society Reviews, Vol. 39, No. 3, 2010, pp. 948-956. doi:10.1039/b900410f

[6] H. Nakamura, Y. Yamaguchi, M. Miyazaki, H. Maeda and M. Uehara, "Preparation of CdSe Nanocrystals in a Micro-Flow-Reactor," Chemical Communications, No. 23, 2002, pp. 2844-2845. doi:10.1039/b208992k

[7] H. Kawazumi, A. Tashiro, K. Ogino and H. Maeda, "Observation of Fluidic Behavior in a Polymethylmethacrylate-Fabricated Microchannel by a Simple Spectroscopic Analysis," Lab on a Chip, Vol. 2, No. 1, 2002, pp. 8-10. doi:10.1039/b109058e

[8] N. Jinno, M. Murakami, K. Mizohata, M. Hashimoto and K. Tsukagoshi, "Fluorescence Observation Supporting Capillary Chromatography Based on Tube Radial Distribution of Carrier Solvents under Laminar Flow Conditions," Analyst, Vol. 136, No. 5, 2011, pp. 927-932. doi:10.1039/c0an00820f

[9] N. Jinno, M. Hashimoto and K. Tsukagoshi, "Experimental Consideration of Capillary Chromatography Based on Tube Radial Distribution of Ternary Mixture Carrier Solvents under Laminar Flow Conditions," Analytical Sciences, Vol. 27, No. 3, 2011, pp. 259-264. doi:10.2116/analsci.27.259

[10] M. Murakami, N. Jinno, M. Hashimoto and K. Tsukagoshi, "Tube Radial Distribution Phenomenon of Ternary Mixed Solvents in a Microspace under Laminar Flow Conditions," Analytical Sciences, Vol. 27, No. 8, 2011, pp. 793-798. doi:10.2116/analsci.27.793

[11] S. Fujinaga, N. Jinno, M. Hashimoto and K. Tsukagoshi, "Use of Tube Radial Distribution of Ternary Mixed Carrier Solvents for Introduction of Absorption Reagent for Metal Ion Separation and Online Detection into Capillary," Journal of Separation Science, Vol. 34, No. 20, 2011, pp. 2833-2839. doi:10.1002/jssc.201100404

[12] N. Jinno, M. Hashimoto and K. Tsukagoshi, "Extraction of $\mathrm{Cu}$ (II) Based on Tube Radial Distribution of Ternary Mixed Carrier Solvent in Microchannels," Chemistry Letters, Vol. 40, No. 6, 2011, pp. 654-655. doi:10.1246/cl.2011.654 